\title{
miR-9 functions as a tumor inhibitor of cell proliferation in epithelial ovarian cancer through targeting the SDF-1/CXCR4 pathway
}

\author{
LIN HE, LI ZHANG, MENGFEI WANG and WENRONG WANG
}

Department of Gynecology, Nanjing Medical University Affiliated Wuxi Second Hospital, Wuxi, Jiangsu 214002, P.R. China

Received April 9, 2015; Accepted May 5, 2016

DOI: $10.3892 /$ etm.2017.4118

\begin{abstract}
The current study aimed to investigate the potential role of miR-9 in the inhibition of ovarian cancer progression through the stromal cell-derived factor-1 (SDF-1)/ C-X-C chemokine receptor type 4 (CXCR4) pathway and to provide a theoretical basis for the diagnosis and treatment of ovarian cancer. Human ovarian cancer OVCAR-3 cells were transfected with miR-9 short hairpin RNA (shRNA). The effect of miR-9 on the mRNA expression levels of CXCR4 were analyzed using reverse transcription-quantitative polymerase chain reaction. The effects of miR-9 on OVCAR-3 cell proliferation, invasion and apoptotic ability were detected using a 3-(4,5-dimethyl-2-thiazolyl)-2,5-diphenyl tetrazolium bromide assay, Matrigel method, and Annexin V-fluorescein isothiocyanate flow cytometry, respectively. In addition, expression levels of SDF-1/CXCR4 pathway associated proteins were determined by western blot analysis. mRNA expression levels of CXCR4 in OVCAR-3 cells transfected with miR-9 shRNA was significantly downregulated compared with the blank and control groups $(\mathrm{P}<0.05)$. Furthermore, compared with the two control groups, the current results revealed that miR-9 inhibited cell proliferation, suppressed invasive ability and induced cell apoptosis in OVCAR-3 cells $(\mathrm{P}<0.05)$. Finally, it was observed that miR-9 functioned as a tumor inhibitor through the SDF-1/CXCR4 pathway by suppressing the expression levels of extracellular signal-regulated kinase 1 (ERK1), ERK2 and matrix metalloproteinase-9 proteins. The present study suggested that miR-9 may function as a promising tumor inhibitor for ovarian cancer through targeting the SDF-1/CXCR4 pathway.
\end{abstract}

Correspondence to: Dr Wenrong Wang, Department of Gynecology, Nanjing Medical University Affiliated Wuxi Second Hospital, 68 Zhongshan Road, Wuxi, Jiangsu 214002, P.R. China E-mail: wangwenrong401@126.com

Key words: ovarian cancer, miR-9, stromal cell-derived factor-1/C-X-C chemokine receptor type 4 pathway, cell proliferation

\section{Introduction}

Ovarian cancer is one of the most common malignancies in women, and may be classified as epithelial ovarian cancer (EOC) and ovarian cancer of germ cell origin $(1,2)$. Statistics indicate that the mortality rate of EOC places it at fourth position among female cancers, and the mortality rates as a result of EOC morbidity have been high in recent years $(3,4)$. Previous studies suggest that it is difficult to diagnose EOC in the early stages in the ovary exists in the specific shady position of pelvis, which also results in a 5-year survival rate of $15-30 \%(5,6)$. Therefore, to explore several key factors for EOC diagnosis in the early stage may be of some use to clinical practice.

MicroRNAs (miRNAs) are a class of endogenous $\sim 22 \mathrm{nt}$ RNAs that serve crucial roles in the human body through targeting mRNAs at the post-transcriptional level (7). Several miRNAs represent a novel class of genes that have important roles as negative regulators of gene expression in a number of diseases in previous studies. For example, Yang et al (8) showed that miR-214 was able to induce cell survival and cisplatin resistance via targeting phosphatase and tensin homolog in ovarian cancer, and Vecchione et al (9) revealed that miR-484 functions independently in ovarian cancer by modulating vascular endothelial growth factor (VEGF) through VEGFB signaling. In addition, several papers have indicated that miR-9 may function as a tumor suppressor in certain types of cancer, such as ovarian cancer and colon cancer $(10,11)$. One such paper by Tang et al (12) determined that miR-9 inhibits cell proliferation, migration and invasion, and is a tumor suppressor in ovarian serous carcinoma through targeting the Talin-1 gene. In addition, cytokine stromal cell-derived factor (SDF-1, also known as CXCL12), a small proinflammatory chemoattractant cytokine that is able to bind to a specific G-protein coupled seven-span transmembrane receptor of CXCR4, is a major regulator of cell trafficking and adhesion (13). Increasing evidence has indicated that the SDF-1/CXCR4 pathway is crucial in promoting tumor cell proliferation and enhancing cell invasion and tumor angiogenesis by activating the downstream signal proteins in variety of tumors, such as ovarian carcinoma, oral cancer and colorectal carcinoma $(14,15)$. Although several papers in the literature have reported the importance of the role of miR-9 in ovarian cancer development, the association between 
miR-9 and the SDF-1/CXCR4 pathway in ovarian cancer proliferation, in addition to its underlying mechanism, have yet to be elucidated.

In the present study, miR-9 was transfected into human ovarian cancer OVCAR-3 cells for the purpose of analyzing the effects of miR-9 in ovarian cancer progression. Comprehensive experimental methods were used to detect the expression levels of CXCR4 mRNA, and to measure the effects of miR-9 on OVCAR-3 cell proliferation, invasion and cell apoptosis. The current study aimed to investigate the potential role of miR-9 in ovarian cancer progression and its potential mechanism, and may provide a theoretical basis for future research concerning ovarian cancer diagnosis and treatment.

\section{Materials and methods}

Cell culture and cell transfection. The human EOC OVCAR-3 cell line (American Type Culture Collecction, Manassas, VA, USA) was cultivated in RPMI 1640 medium (Invitrogen; Thermo Fisher Scientific, Inc., Waltham, MA, USA) supplemented with $20 \%$ fetal bovine serum (FBS; Sigma-Aldrich, St. Louis, MO, USA) and incubated at $37^{\circ} \mathrm{C}$ in 5\% $\mathrm{CO}_{2}$. The miR-9 mimics were purchased from Sangon Biotech Co., Ltd. (Shanghai, China), and synthetic small duplex sequences of miR-9-RNA were bioprocessed into mature miR-9 in cells. Total cells were separated into three groups as follows: i) Blank (cells transfected without miR-9 RNA); ii) negative control (NC) sequence was used to eliminate any potential non-sequence specific effects; and iii) experimental group (cells transfected with miR-9 RNA). Briefly, OVCAR-3 cells in the logarithmic phase were transferred onto 6-well plates, followed by the transfection of miR-9 mimics into cells according to the protocol supplied with the Lipofectamine 2000 (Invitrogen; Thermo Fisher Scientific, Inc.). Subsequently, cells were cultivated using RPMI-1640 medium without antibiotics, and Lipofectamine 2000 and miR-9 mimics were diluted with serum-free medium. Primers were as follows: Sense, 5'-GGG TCTTTGGTTATCTAGC-3' and antisense, 5'-TGCGTGTCG TGGAGTC-3' for miR-9 amplification; and sense, 5'-UUC UCCGAACGUGUCACGUTT-3' and antisense, 5'-ACGUGA CACGUUCGGAGAATT-3' for the silenced miR-9 vector construction.

Reverse transcription-quantitative polymerase chain reaction (RT-qPCR). The OVCAR-3 cells in three groups collected at $48 \mathrm{~h}$ underwent grinding in liquid nitrogen and were then washed with PBS buffer ( $\mathrm{pH}$ 7.4). The total RNA from OVCAR-3 cells was isolated using TRIzol extraction reagent (Invitrogen; Thermo Fisher Scientific, Inc.) according to the manufacturer's protocol (16), and the isolated RNA was treated with RNase-free Dnase I (Promega Corporation, Madison, WI, USA) to remove genomic DNA. The concentration and purity of extracted RNA was detected using SMA4000 UV-VIS (Merinton Instrument, Inc., Ann Arbor, MI, USA). The purified RNA $(0.5 \mu \mathrm{g} / \mu \mathrm{l})$ was used for cDNA synthesis with the PrimerScript 1st Strand cDNA Synthesis kit (Takara Biotechnology Co., Ltd., Dalian, China). Primers used for target amplification were as follows: Sense, 5'-CTTCTTAAC TGGCATTGTGG-3' and antisense, 5'-ACTGAACCTGAC CGTACAGTGATGACAAAG-3'. RT-qPCR was performed using an Eppendorf Mastercycler (Brinkmann Instruments, Westbury, NY, USA) using the SYBR ExScript RT-qPCR Kit (Takara Biotechnology Co., Ltd.). A total reaction system of $20 \mu \mathrm{l}$ volume contained $1 \mu \mathrm{l} \mathrm{cDNA}$ from the aforementioned PCR, $10 \mu 1$ SYBR Premix EX Taq, $1 \mu$ l of each of the primers $(10 \mu \mathrm{M})$, and $7 \mu \mathrm{ldd} \mathrm{H}_{2} \mathrm{O}$. The PCR program was as follows: Denaturation at $95^{\circ} \mathrm{C}$ for $2 \mathrm{~min}$; followed by 45 cycles at $95^{\circ} \mathrm{C}$ for $10 \mathrm{sec}, 59^{\circ} \mathrm{C}$ for $20 \mathrm{sec}$ and $72^{\circ} \mathrm{C}$ for $30 \mathrm{sec}$. The $2^{-\Delta \Delta \mathrm{CT}}$ method was used to determine the relative gene expression levels. Melting curve analysis of amplification products was performed at the climax of each PCR to confirm that only one product was amplified and detected. GAPDH was used as the internal control.

3-(4,5-dimethyl-2-thiazolyl)-2,5-diphenyl tetrazolium bromide (MTT) assay. Cell survival rate and sensitivity of OVCAR-3 tumor cells at different time points following transfection with miR-9 or NC vectors were detected using MTT assay as previously described (17). Briefly, $200 \mu 1$ OVCAR-3 tumor cells in the logarithmic phase were transfected into 24-well plates with RPMI 1640 medium supplemented with $10 \%$ FBS for $24 \mathrm{~h}$. Each assay was performed six times and repeated three times independently. Subsequently, MTT (5 mg/ml; Sigma-Aldrich) was added into the 24-well plates to combine with tumor cells, and incubated at $37^{\circ} \mathrm{C}$ for $4 \mathrm{~h}$. Mixtures were centrifuged at $1,000 \mathrm{x}$ g for $10 \mathrm{~min}$, followed by removal of the supernatant. Next, $150 \mu$ l dimethyl sulfoxide was added to the harvested cells to dissolve the formazan. The absorbance of cells in each plate was measured at $570 \mathrm{~nm}$ using a microplate reader (THERMOMultiskan; M3, Fort Washington, PA, USA).

Cell invasion assay. The invasive ability of OVCAR-3 cells was detected on Matrigel-coated $(0.8 \mathrm{mg} / \mathrm{ml})$ Transwell inserts with $8 \mu \mathrm{m}$ pore size (BD Biosciences, Franklin Lakes, NJ, USA). The miR-9 RNA and scrambled OVCAR-3 cells were starved for $24 \mathrm{~h}$, then harvested. Subsequent to this, cells (300 $\mu \mathrm{l}$; cell density, $1 \times 10^{5}$ cells/ml) were seeded into the upper well of the chamber containing serum free medium. The lower chamber contained RPMI 1640 with 10\% BSA. Following an incubation period of $12 \mathrm{~h}$, cells were invaded onto the membrane and fixed with $70 \%$ ethanol and stained with $0.1 \%$ crystal violet and sealed on slides. The number of cells across the membrane was calculated using a light microscope.

Cell apoptosis assay. Effect of miR-9 on OVCAR-3 tumor cell apoptosis was quantified by flow cytometry using Annexin V-fluorescein isothiocyanate (FITC) cell apoptosis kit (Invitrogen; Thermo Fisher Scientific, Inc.) according to manufacturer's protocol (18). Briefly, the OVCAR-3 cells were transfected with miR-9 or NC vectors for $36 \mathrm{~h}$, followed by the replacement of cell culture medium with serum-free RPMI 1640 medium. Total cells were harvested and washed using PBS buffer ( $\mathrm{pH}$ 7.4) three times, and resuspended in the staining buffer provided in the kit. Following this, $5 \mu 1$ Annexin V-FITC and $5 \mu \mathrm{l}$ propidium iodide (PI) were mixed with the cells. After being cultivated at room temperature for $10 \mathrm{~min}$, mixtures were analyzed using the FACScan flow cytometry (BD Biosciences). Annexin V-positive and PI-negative cells were considered to be apoptotic cells. 
Western blot analysis. OVCAR-3 tumor cells at $48 \mathrm{~h}$ were lysed in radioimmunoprecipitation assay (Sangon Biotech Co., Ltd.) lysate containing phenylmethanesufonyl fluoride and centrifuged at $8,000 \times \mathrm{g}$ for $10 \mathrm{~min}$ at $4^{\circ} \mathrm{C}$. Supernatant was collected to determine the concentration of proteins using a bicinchoninic acid protein assay kit (Pierce Biotechnology, Inc., Rockford, IL, USA). A total of $25 \mu \mathrm{g}$ protein per cell lysates was then subjected to $10 \%$ SDS-PAGE and transferred onto a polyvinylidene fluoride membrane (Merck KGaA, Darmstadt, Germany). The membrane was blocked in Tris-buffered saline/Tween 20 (TBST) with 5\% non-fat milk for $1 \mathrm{~h}$, and subsequently incubated with rabbit anti-human extracellular signal-regulated kinase 1 (ERK1; ab17942), ERK2 (ab32081) or matrix metalloproteinase-9 (MMP-9; ab38898; all Abcam, Cambridge, UK) monoclonal antibodies (1:100) overnight at $4^{\circ} \mathrm{C}$, then incubated with horseradish peroxidase labeled goat anti-rat secondary antibody $(1: 1,000 ;$ ab7010; Abcam) at room temperature for $1 \mathrm{~h}$. Subsequently, the membrane was washed using the X1 TBST buffer for 10 min three times. Detection was performed using the development of X-ray after chromogenic substrate with an enhanced chemiluminescence method. In addition, $\beta$-actin (Sigma-Aldrich) served as the internal control.

Statistical analysis. All data are expressed as the mean \pm standard error of the mean. Independent sample t-test was used to calculate the difference among groups (Blank compared with control; miR-9 compared with Blank, and miR-9 compared with control) using GraphPad Prism software (version 5.0; GraphPad Software, Inc., San Diego, CA, USA). $\mathrm{P}<0.05$ was defined as statistically significant.

\section{Results}

miR-9 inhibited CXCR4 expression in ovarian tumor cells. In order to analyze the effect of miR-9 on CXCR4 expression levels in OVCAR-3 tumor cells, shRNA vectors of miR-9 or $\mathrm{NC}$ were transfected into OVCAR-3 cells (Fig. 1). The results indicated that CXCR4 expression levels in OVCAR-3 cells that were transfected with miR-9 declined significantly compared with those in the control group $(\mathrm{P}<0.05)$, other than this, there was no significant difference in CXCR4 expression levels in the Blank group compared with the NC group $(\mathrm{P}>0.05)$.

MTT assay. The effect of miR-9 on ovarian tumor OVCAR-3 cell vitality was detected using an MTT assay (Fig. 2). The results indicated that there was no significant difference in cell vitality between the Blank and $\mathrm{NC}$ groups with increasing time $(\mathrm{P}>0.05)$. However, cell vitality in the miR-9 group was significantly decreased compared with that in the Blank and $\mathrm{NC}$ groups with increasing time $(\mathrm{P}<0.05)$, indicating that miR-9 may inhibit OVCAR-3 cell vitality.

Cell invasion assay. The cell invasive ability of OVCAR-3 cells transfected with miR-9 was assessed using the Matrigel method (Fig. 3). There was no significant difference in cell invasive ability between the Blank and control groups ( $P>0.05)$, however, the cell number of OVCAR-3 cells in the miR-9 group was significantly declined compared with the Blank and NC groups $(\mathrm{P}<0.05)$, suggesting that miR-9 may

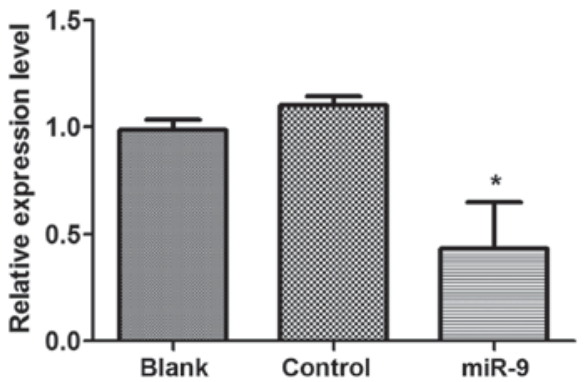

Figure 1. Reverse transcription-quantitative polymerase chain reaction analysis of relative CXCR4 expression levels in OVCAR-3 cells. " $\mathrm{P}<0.05$ vs. Blank and Control groups.

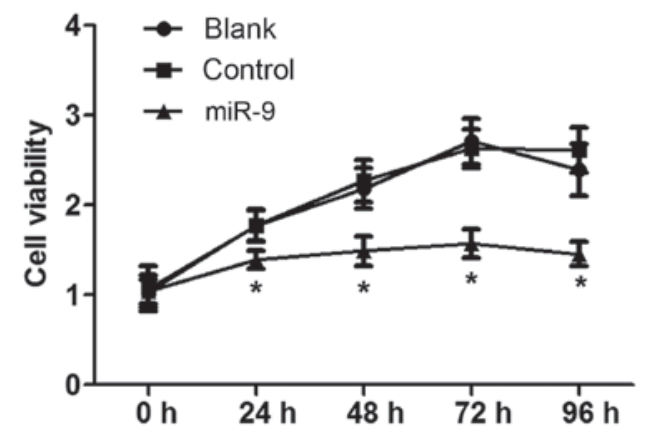

Figure 2. MTT assay examining cell viability of OVCAR-3 cells in 24-h increments. " $\mathrm{P}<0.05$ vs. Blank and Control groups.

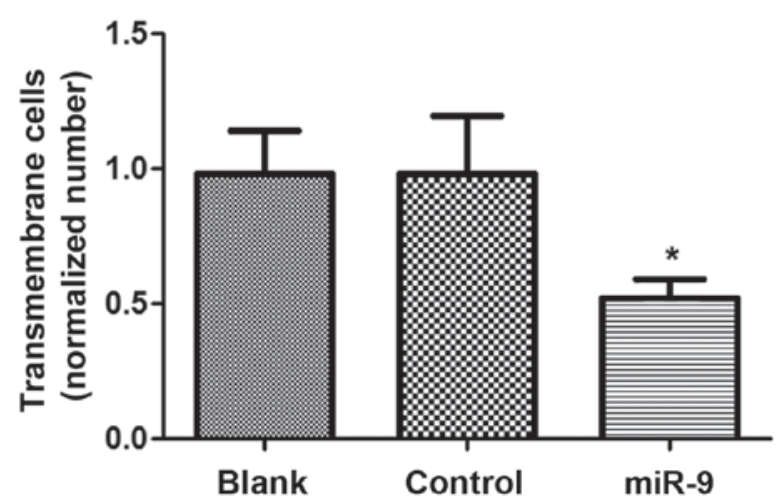

Figure 3. Invasion analysis of OVCAR-3 cells in Blank, control and miR-9 treated groups. ${ }^{*} \mathrm{P}<0.05$ vs. Blank and Control groups.

suppress the invasive ability of ovarian tumor OVCAR-3 cells.

Cell apoptosis. In order to assess the effect of miR-9 on OVCAR-3 cell apoptosis, Annexin V was used to measure the apoptotic cells (Fig. 4). The results revealed that the apoptotic cells in the Blank and NC groups were 1.43 and 2.86\%, respectively (Fig. 4A and B). The amount of apoptotic cells in the miR-9 group was $4.5 \%$ (Fig. 4C), implying that miR-9 may accelerate OVCAR-3 cell apoptosis.

Western blot analysis. To identify the association between miR-9 with the SDF-1/CXCR4 signal pathway in ovarian cancer, western blot analysis was conducted to detect the 

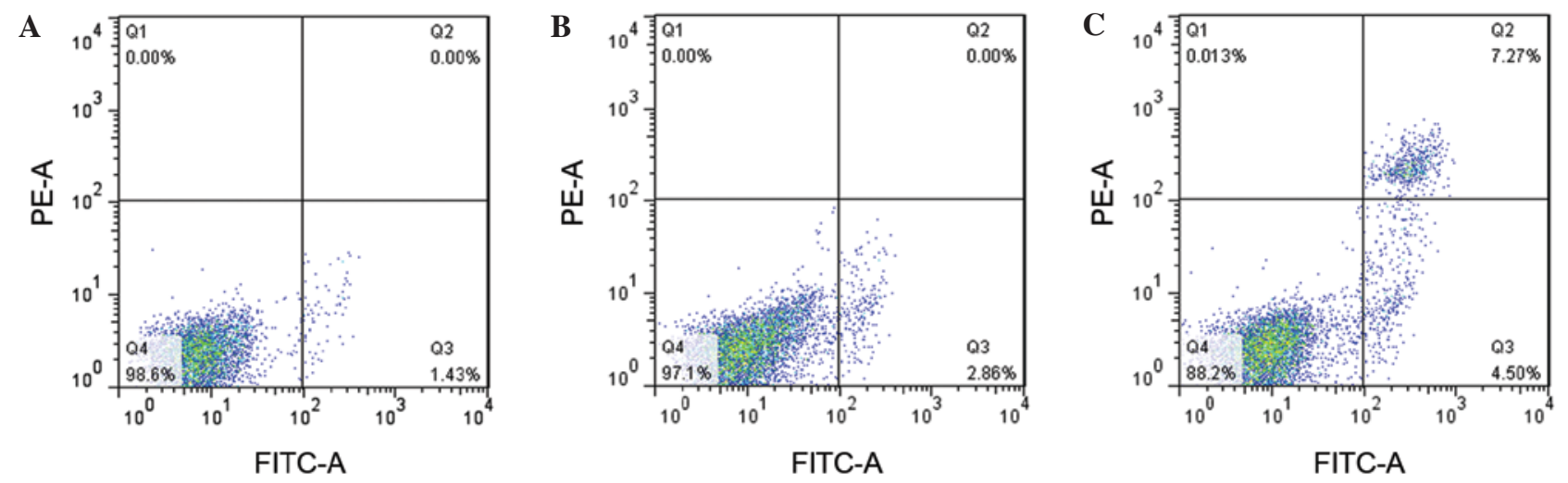

Figure 4. Cell apoptosis analysis of OVCAR-3 cells in three groups, (A) Blank, (B) control and (C) mir-9. B, and C stands for the Blank, control, and miR-9 groups. FITC-A, Annexin V-fluorescein isothiocyanate.
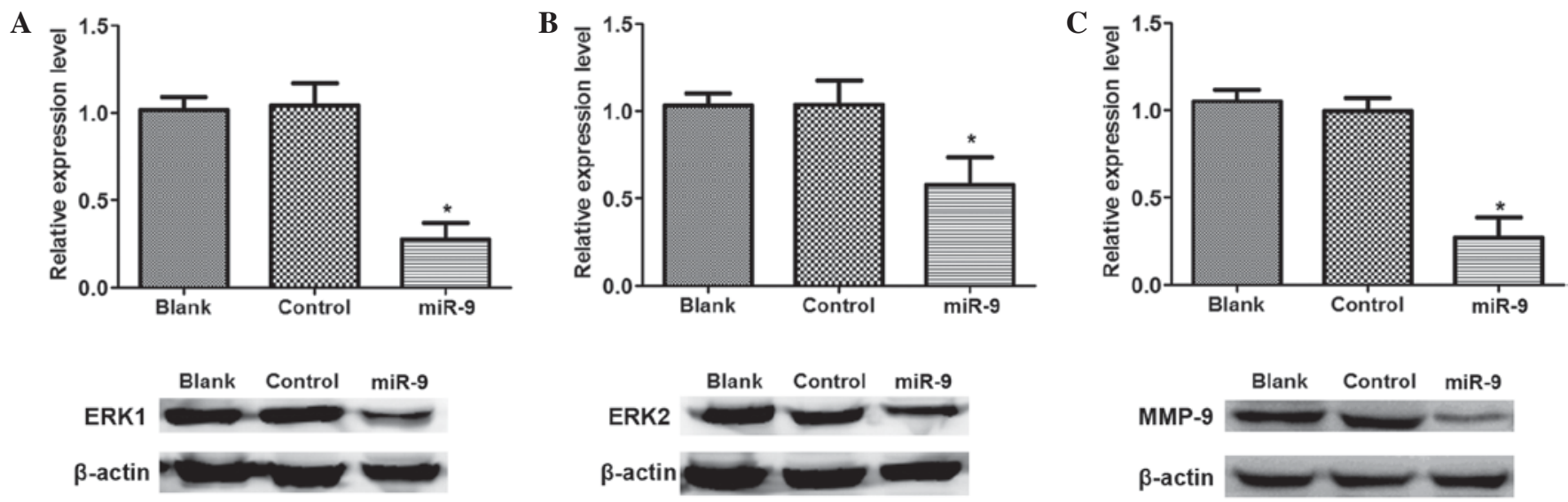

Figure 5. Western blot analysis of SDF-1/CXCR4 signaling pathway associated proteins. Relative expression levels of (A) ERK1 protein in the three groups; (B) ERK2 protein in three groups; (C) MMP-9 protein in three groups. " $\mathrm{P}<0.05$ vs. Blank and Control groups. ERK, extracellular signal-regulated kinase.

signal pathway associated proteins (Fig. 5). There was no significant difference in ERK1, ERK2 and MMP-9 protein expression levels between Blank and control group $(\mathrm{P}>0.05$; Fig. 5). However, the expression levels of ERK1, ERK2 and MMP-9 in miR-9 groups were significantly reduced compared with those in the Blank or $\mathrm{NC}$ groups $(\mathrm{P}<0.05)$.

\section{Discussion}

Ovarian cancer is one of the most prominent malignancies detected in females worldwide $(1,2)$, and the mortality rates associated with EOC has led it to be ranked fourth among female cancers, and mortality resulting from EOC morbidity has been particularly high in recent years $(3,4)$. Despite the importance of the role of miR- 9 in ovarian cancer development being widely reported in the literature $(10,12)$, the association between miR-9 and the SDF-1/CXCR4 pathway in ovarian cancer proliferation and its potential mechanism have yet to be fully elucidated. In the present study, the effect of miR-9 on ovarian cancer cell proliferation was analyzed by transfecting miR-9 RNA into OVCAR-3 cells. The current data indicated that miR-9 was able to downregulate the mRNA expression levels of CXCR4, inhibit cell proliferation, suppress cell invasion and induce cell apoptosis of OVCAR-3 cells. Furthermore, miR-9 was able to downregulate the protein expression levels of ERK1, ERK2 and MMP-9 in OVCAR-3 cells.

The current data displayed that miR-9 was able to inhibit cell proliferation, suppress invasive ability and induce cell apoptosis of OVCAR-3 cells. Prior studies have demonstrated that inhibiting cell proliferation, suppressing cell invasion and inducing cell apoptosis could block tumor metastasis $(19,20)$. Laios et al (11) demonstrated that miR-9 blocked cell proliferation and angiogenesis, induced cell apoptosis and repressed invasion in ovarian cancer. Additionally, Guo et al (21) revealed that cell growth was inhibited by miR-9 through NF-kB1 signaling in ovarian cancer. In addition, Tan et al (22) established that miR-9 reduced cell invasion in SK-Hep-1 cells, consequently suppressing tumor metastasis. In a study by Lujambio et al (23), the methylation status of miR-9 was certified to be associated with cancer metastasis by influencing biological processes such as cell apoptosis, differentiation and proliferation. Based on the current results, we hypothesize that miR-9 may function to suppress ovarian cancer metastasis by influencing cell invasion, proliferation and apoptosis.

Furthermore, the current results revealed that CXCR4 was downregulated by miR-9 in OVCAR-3 cells. CXCR4 is the receptor for SDF-1 (CXCL12 cytokine) and is involved in cancer progression through certain signals (24). It has 
been demonstrated that endocrine disrupting chemicals are able to promote cell growth in ovarian cancer through the CXCL-12-CXCR4 signaling pathway (25). In addition, Popple et al (26) revealed that SDF-1 was able to induce the proliferation of tumor cells and thus may be an independent predictor of poor survival in ovarian cancer. Furthermore, Lu et al (27) identified that miR-9 acted as a tumor suppressor in nasopharyngeal carcinoma by repressing CXCR4 expression, and miR-9 has been determined to be an inhibitor on cell proliferation in oral squamous cell carcinoma by suppressing CXCR4 expression (28). Conversely, the expression levels of ERK1, ERK2 and MMP-9 were also downregulated by miR-9 in this study. The activated ERK1/2 was able to induce CXCR4 expression in cancers (29) and Yu et al (30) found that CXCR4 enhanced squamous cell carcinoma migration and invasion by inducing MMP-9 expression through the ERK signaling pathway. MMP-9 serves a crucial role in tumor invasion and metastasis (31), and it has been reported that SDF-1 enhances cell invasion of ovarian cancer by upregulating MMP-9 expression (32). In accordance with previous results, CXCR4 and MMP-9, ERK1/2 expressions were lower in the miR-9 group compared with the controls, indicating that miR-9 may be a suppressor of ovarian cancer metastasis by downregulating the SDF-1/CXCR4 pathway by suppressing ERK1/2 and MMP-9 expression levels.

In conclusion, the current study investigated the effect of miR-9 in ovarian cancer metastasis and investigated potential underlying mechanisms. Overall, the current study ascertained that miR-9 downregulates CXCR4 expression in ovarian cancer OVCAR-3 cells and inhibits OVCAR-3 cell proliferation and suppresses cell invasiveness. In addition, miR-9 induces OVCAR-3 cell apoptosis and is a tumor inhibitor for ovarian cancer via the SDF-1/CXCR4 pathway. Thus, miR-9 may function as a promising tumor therapy by inhibiting cell proliferation, suppressing invasion and inducing cell apoptosis via the downregulation of the SDF-1/CXCR4 pathway in ovarian cancer. The present study may provide a basis for future research pertaining to the diagnosis of ovarian cancer. However, further experimental studies are required to explore the complex mechanisms underlying the involvement of miR-9 in ovarian cancer.

\section{References}

1. Miow QH, Tan TZ, Ye J, Lau JA, Yokomizo T, Thiery JP and Mori S: Epithelial-mesenchymal status renders differential responses to cisplatin in ovarian cancer. Oncogene 34: 1899-1907, 2015.

2. Nik NN, Vang R, Shih IeM and Kurman RJ: Origin and pathogenesis of pelvic (ovarian, tubal and primary peritoneal) serous carcinoma. Ann Rev Pathol 9: 27-45, 2014.

3. Marcus CS, Maxwell GL, Darcy KM, Hamilton CA and McGuire WP: Current approaches and challenges in managing and monitoring treatment response in ovarian cancer. J Cancer 5: 25-30, 2014.

4. Siegel R, Ma J, Zou Z and Jemal A: Cancer statistics, 2014. CA Cancer J Clin 64: 9-29, 2014.

5. Tone AA, McConechy MK, Yang W, Ding J, Yip S, Kong E, Wong KK, Gershenson DM, Mackay H, Shah S, et al: Intratumoral heterogeneity in a minority of ovarian low-grade serous carcinomas. BMC Cancer 14: 982, 2014.

6. Dillman RO, DePriest C, Ellis R and de Leon C: 5-year survival for patients with metastatic melanoma who had no evidence of disease at time of treatment with patient specific tumor stem cell vaccines. Cancer Res 74 (Suppl 19): 197, 2014.
7. Bartel DP: MicroRNAs: Genomics, biogenesis, mechanism and function. Cell 116: 281-297, 2004.

8. Yang H, Kong W, He L, Zhao JJ, O'Donnell JD, Wang J, Wenham RM, Coppola D, Kruk PA, Nicosia SV and Cheng JQ: MicroRNA expression profiling in human ovarian cancer: miR-214 induces cell survival and cisplatin resistance by targeting PTEN. Cancer Res 68: 425-433, 2008.

9. Vecchione A, Belletti B, Lovat F, Volinia S, Chiappetta G, Giglio S, Sonego M, Cirombella R, Onesti EC, Pellegrini P, et al: A microRNA signature defines chemoresistance in ovarian cancer through modulation of angiogenesis. Proc Natl Acad Sci USA 110: 9845-9850, 2013.

10. Cekaite L, Rantala JK, Bruun J,Guriby M, Agesen TH,Danielsen SA, Lind GE, Nesbakken A, Kallioniemi O, Lothe RA and Skotheim RI: MiR-9, -31, and -182 deregulation promote proliferation and tumor cell survival in colon cancer. Neoplasia 14: 868-879, 2012.

11. Laios A, O'Toole S, Flavin R, Martin C, Kelly L, Ring M, Finn SP, Barrett C, Loda M, Gleeson N, et al: Potential role of miR-9 and miR-223 in recurrent ovarian cancer. Mol Cancer 7: 35, 2008.

12. Tang H, Yao L, Tao X, Yu Y, Chen M, Zhang R and Xu C: miR-9 functions as a tumor suppressor in ovarian serous carcinoma by targeting TLN1. Int J Mol Med 32: 381-388, 2013.

13. Teicher BA and Fricker SP: CXCL12 (SDF-1)/CXCR4 pathway in cancer. Clin Cancer Res 16: 2927-2931, 2010.

14. Yu Y, Shi X, Shu Z, Xie T, Huang K, Wei L, Song H, Zhang W and Xue X: Stromal cell-derived factor-1 (SDF-1)/CXCR4 axis enhances cellular invasion in ovarian carcinoma cells via integrin $\beta 1$ and $\beta 3$ expressions. Oncol Res 21: 217-225, 2013.

15. Kinouchi M, Uchida D, Kuribayashi N, Tamatani T, Nagai H and Miyamoto Y: Isolation of a novel metastasis-related microRNA, miR-518c-5p, induced by the stromal cell-derived factor (SDF)-1/CXCR4 system in oral cancer. Cancer Res 74 (Suppl 19): 1446, 2014

16. Hummon AB, Lim SR, Difilippantonio MJ and Ried T: Isolation and solubilization of proteins after TRIzol extraction of RNA and DNA from patient material following prolonged storage. Biotechniques 42: 467-470, 2007.

17. Szotek PP, Pieretti-Vanmarcke R, Masiakos PT, Dinulescu DM, Connolly D, Foster R, Dombkowski D, Preffer F, Maclaughlin DT and Donahoe PK: Ovarian cancer side population defines cells with stem cell-like characteristics and mullerian inhibiting substance responsiveness. Proc Natl Acad Sci USA 103: 11154-11159, 2006.

18. Shirali S, Aghaei M, Shabani M, Fathi M, Sohrabi M and Moeinifard M: Adenosine induces cell cycle arrest and apoptosis via cyclinD1/Cdk4 and Bcl-2/Bax pathways in human ovarian cancer cell line OVCAR-3. Tumor Biol 34: 1085-1095, 2013.

19. Zetter BR: Angiogenesis and tumor metastasis. Ann Rev Med 49: 407-424, 1998

20. Steeg PS: Tumor metastasis: Mechanistic insights and clinical challenges. Nat Med 12: 895-904, 2006.

21. Guo LM, Pu Y, Han Z, Liu T, Li YX, Liu M, Li X and Tang H: MicroRNA-9 inhibits ovarian cancer cell growth through regulation of NF-kappaB1. FEBS J 276: 5537-5546, 2009.

22. Tan HX, Wang Q, Chen LZ, Huang XH, Chen JS, Fu XH, Cao LQ, Chen XL, Li W and Zhang LJ: MicroRNA-9 reduces cell invasion and E-cadherin secretion in SK-Hep-1 cell. Med Oncol 27: 654-660, 2010.

23. Lujambio A, Calin GA, Villanueva A, Ropero S, Sánchez-Céspedes M, Blanco D, Montuenga LM, Rossi S, Nicoloso MS, Faller WJ, et al: A microRNA DNA methylation signature for human cancer metastasis. Proc Natl Acad Sci USA 105: 13556-13561, 2008.

24. Obermajer N, Muthuswamy R, Odunsi K, Edwards RP and Kalinski P: PGE2-induced CXCL12 production and CXCR4 expression controls the accumulation of human MDSCs in ovarian cancer environment. Cancer Res 71: 7463-7470, 2011.

25. Hall JM and Korach KS: Endocrine disrupting chemicals promote the growth of ovarian cancer cells via the ER-CXCL12-CXCR4 signaling axis. Mol Carcinog 52: 715-725, 2013.

26. Popple A, Durrant L, Spendlove I, Rolland P, Scott IV, Deen S and Ramage JM: The chemokine, CXCL12, is an independent predictor of poor survival in ovarian cancer. Br J Cancer 106: 1306-1313, 2012.

27. Lu J, Luo H, Liu X, Peng Y, Zhang B, Wang L, Xu X, Peng X, Li G, Tian W, et al: miR-9 targets CXCR4 and functions as a potential tumor suppressor in nasopharyngeal carcinoma. Carcinogenesis 35: 554-563, 2014.

28. Yu T, Liu K, Wu Y, Fan J, Chen J, Li C, Yang Q and Wang Z: MicroRNA-9 inhibits the proliferation of oral squamous cell carcinoma cells by suppressing expression of CXCR 4 via the Wnt/ $\beta$-catenin signaling pathway. Oncogene 33: 5017-5027, 2014. 
29. Hatano K, Yamaguchi S, Nimura K, Murakami K, Nagahara A, Fujita K, Uemura M, Nakai Y, Tsuchiya M, Nakayama M, et al: Residual prostate cancer cells after docetaxel therapy increase the tumorigenic potential via constitutive signaling of CXCR4, ERK1/2 and c-Myc. Mol Cancer Res 11: 1088-1100, 2013.

30. Yu T, Wu Y, Helman JI, Wen Y, Wang C and Li L: CXCR4 promotes oral squamous cell carcinoma migration and invasion through inducing expression of MMP-9 and MMP-13 via the ERK signaling pathway. Mol Cancer Res 9: 161-172, 2011.
31. Roomi M, Monterrey J, Kalinovsky T, Rath M and Niedzwiecki A: In vitro modulation of MMP-2 and MMP-9 in human cervical and ovarian cancer cell lines by cytokines, inducers and inhibitors. Oncol Rep 23: 605-614, 2010.

32. Yu Y, Li H, Xue B, Jiang X, Huang K, Ge J, Zhang H and Chen B: SDF-1/CXCR7 axis enhances ovarian cancer cell invasion by MMP-9 expression through p38 MAPK pathway. DNA Cell Biol 33: 543-549, 2014. 